\title{
Study on Graphene-modified FRP Bars Used in the Process of Construction Health Management
}

\author{
Xiuzhi Huang ${ }^{1,}$, Jiahui Zhang ${ }^{1}$ \\ ${ }^{1}$ Civil Engineering Department, Southeast University, China \\ ahuangxiuzhi9@gmail.com
}

\begin{abstract}
The distributed long-gauge optical sensor on fiber reinforced polymer (FRP) bar cannot be manufactured through integrated production in current situation, which is hard for construction management. On the other hand, the point-sensing technology of the self-sensing bar will cause deviations in structural health monitoring (SHM). To solve these issues, applying the graphene/epoxy on FRP members is a feasible method for the piezoresistive characteristics of graphene. In this paper, basalt FRP (BFRP) bars with graphene/epoxy film were tested under repeated and cyclic loads and the resistance was measured at the same time until they were broken down. The results suggested that the test pieces can maintain stable sensing performance under repeated and cyclic loads. These facts indicated that the graphene-modified BFRP bars can well reflect the stress condition of the structural member within a safe range and can maintain stable self-sensing performance in the construction health management.
\end{abstract}

Keywords: BFRP bars, Construction management, Fatigue test, Graphene-modification.

\section{Introduction}

FRP composite materials have excellent properties such as lightweight, high strength, corrosion resistance, fatigue resistance, and creep resistance [1-2]. At present, the health monitoring technologies used for bars can be divided into distributed long-gauge Fiber Bragg Grating (FBG) sensing technology and self-sensing bars. Figure 1. is a diagram of damage identification in long-gauge FBG applied to FRP bar. This strain sensor uses the distributed strain measurement technology and realizes high resolution with long- gauge length based on optical fiber technology, which overcomes the deficiencies of traditional overall sensing and local sensing. It has realized the SHM of the key areas and the identification of the structure characteristics at the same time. Wu et al [3-4] have developed a long-gauge FBG strain sensor based on the principle of distributed sensing. The basic sensor performance was evaluated based on the mechanics and perception performance of the FRP-OFBG composite smart bar. However, in the current situation, most of the long-gauge sensor research's method is to stick the sensor on the surface of the measured body, which cannot complete the requirement of cable integration. Most self-monitoring cables use pointtype sensing technology, which can easily cause measurement deviations. Therefore, it is particularly important to complete the precise monitoring of health performance based on the production of integrated cables.

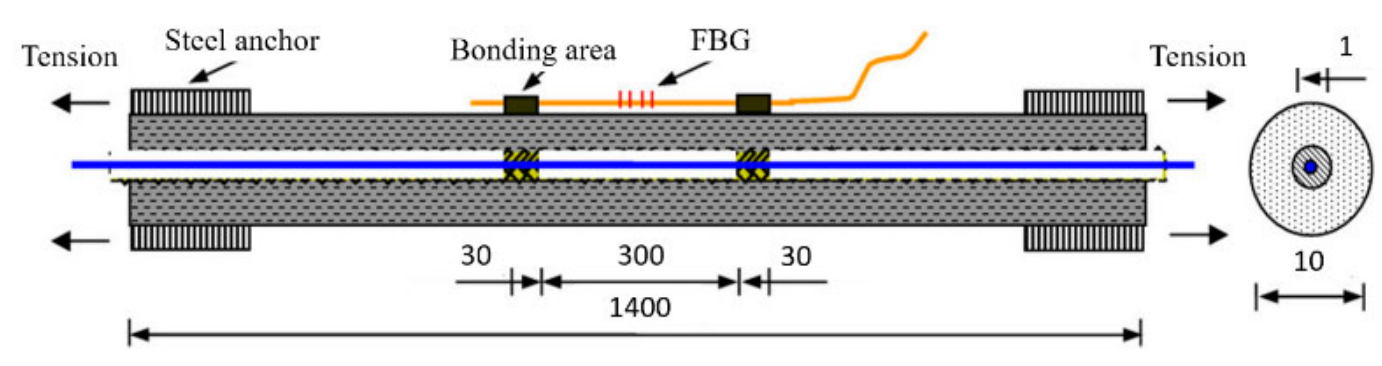

Figure 1. Long gauge length FBG applied to FRP bar damage identification (unit: $\mathrm{m}$ )

With the development of the miniaturization and weight reduction of sensor devices, the graphene, which is the most conductive material, has attracted widespread attention. Due to the piezoresistive properties of graphene, it can form a conductive network in the polymer to achieve the selfinductance of the polymer while reinforcing the polymer at the same time. This is attributed to its large specific surface area and excellent carrier mobility, and it is easy to form a stable conductive network, thereby improving the conductivity of the original composite material and increasing its functionality [5-6]. When the graphene conductive agent is added to the resin matrix, the graphene is uniformly dispersed, and the free graphene nanoplatelets overlap with each other to form a stable conductive network as its concentration gradually increases [7], which is shown in Figure 2. Since the BFRP bar itself is not self-sensing, it is considered to apply a graphene-modified resin with sensing properties to the BFRP bar to realize the need for its effective self-sensing function. In construction situation, the cable or bar which is used in reality will undertake cyclic load frequently. In this paper, the fatigue experiment is taken to testify the mechanical property of the graphene-modified BFRP bar under realistic traffic conditions. 


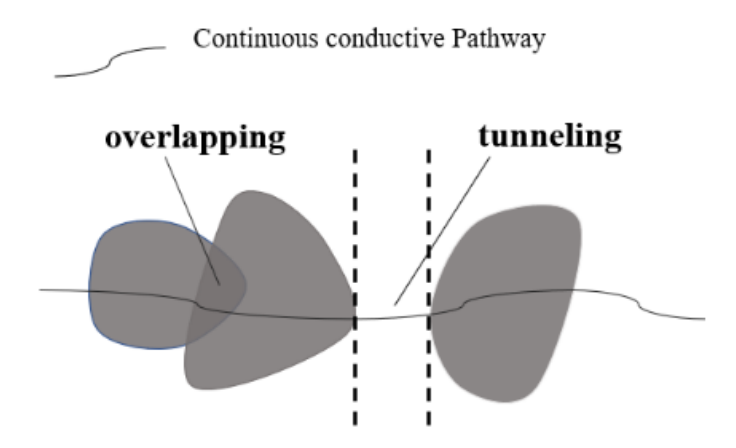

Figure 2. Modeling of distribution of graphene platelets in epoxy

\section{Specimen and Experiment System}

BFRP bars $(1000 \times 35 \times 6 \mathrm{~mm})$ were built in the laboratory and dried for two days referring to the requirements of the American standard ACI.440.3R-04. The anchorage length was $300 \mathrm{~mm}$ at both ends, as shown in Figure 3.

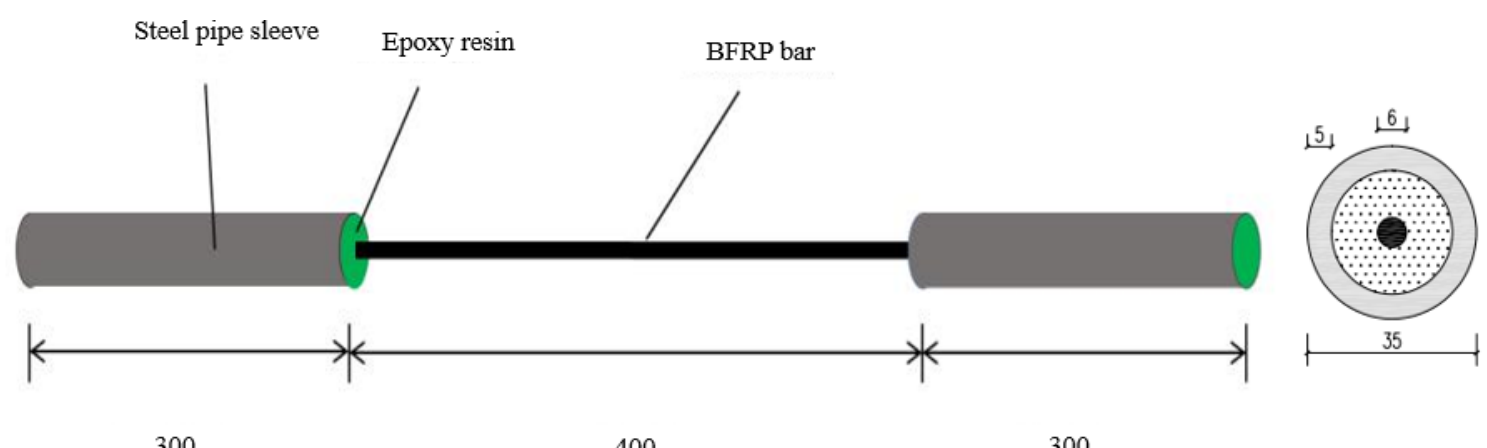

Figure 3. Schematic diagram of bonding steel pipe anchoring

The static tensile experiment is conducted under the Swiss LFV-1000 hydraulic servo fatigue testing machine (as shown in Figure 4.). The maximum load is $\pm 900 \mathrm{kN}$, the load accuracy is $0.01 \mathrm{kN}$, and the displacement accuracy is $0.001 \mathrm{~mm}$. The experiment adopts force-controlled loading with a speed of $0.08 \mathrm{kN} / \mathrm{s}$ to ensure dense sampling during the loading process. The strain measurement equipment is the
TDS-530 static collection instrument, which is set to collect data per second. The strain data is the average value of the real-time data corresponding to the two strain gauges on the specimen. The Amber AT516 DC resistance tester is used for the resistance measurement. The test system is shown in Figure 5.
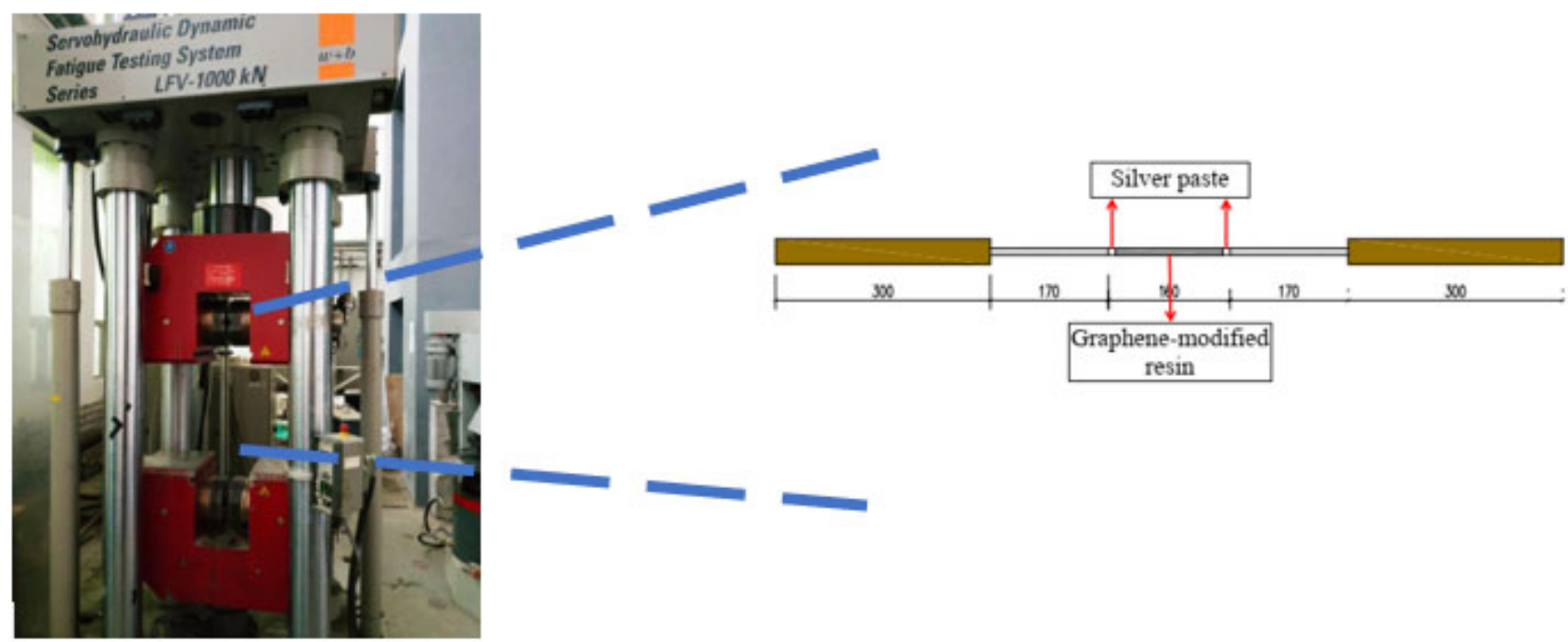

Figure 4. Test loading device and structure test piece (unit: $\mathrm{mm}$ ) 


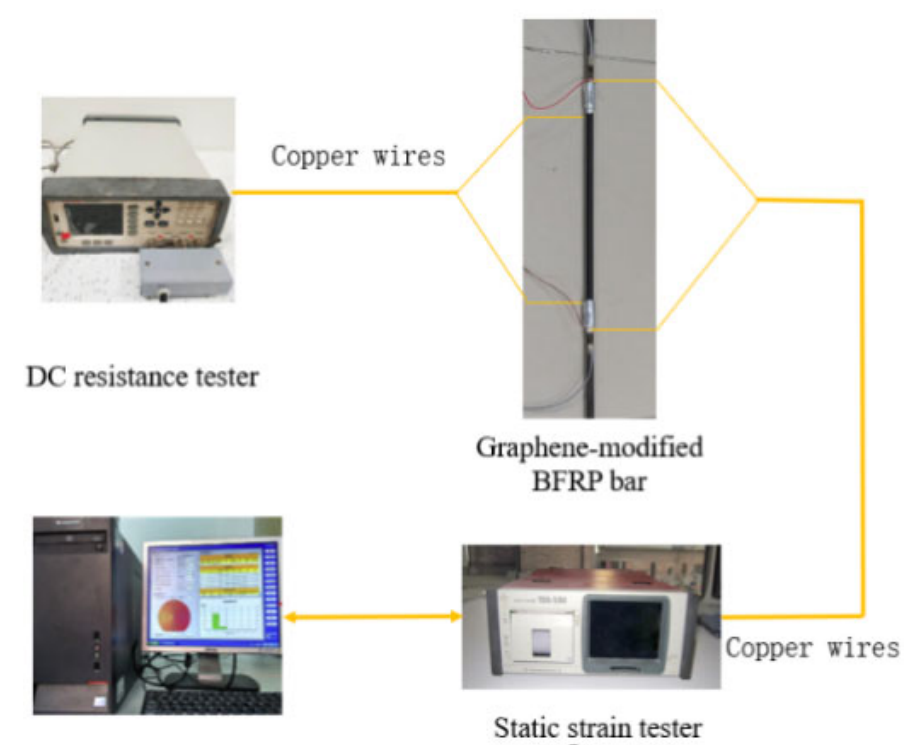

Figure 5. Schematic view of the experiment system

\section{Experiment Results and Discussion}

\subsection{The Effect of Repeated Loading on Sensing Performance}

The purpose of the self-monitoring BFRP bar under

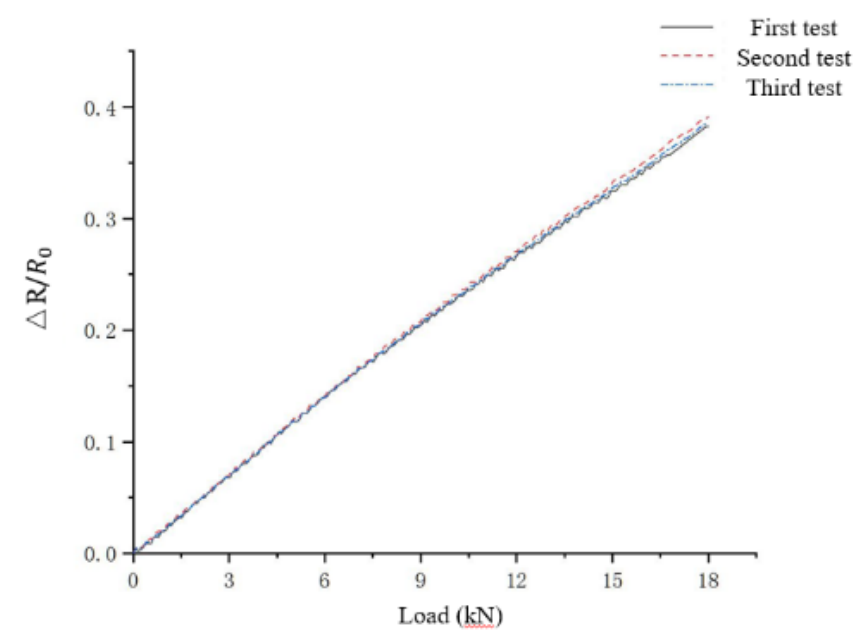

(a) repeatable static load test is to verify that its sensing performance is still effective, which is particularly important when the bar is repeatedly subjected to vehicle loads in actual projects. The resistance change rate-load curve during three repeated measurements is shown in Figure 6.

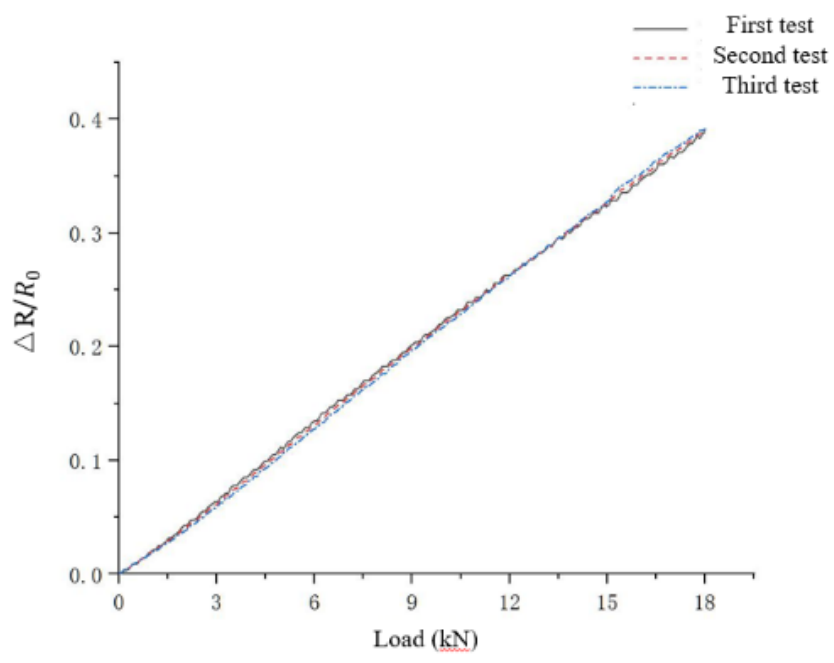

(b)

Figure 6. Repeated load test performance results (a) Specimen 1(b) Specimen 2

$$
K_{\varepsilon}=\frac{\Delta R / R_{0}}{\Delta \varepsilon}=\frac{\Delta R / R_{0}}{\Delta F} \times \frac{\Delta F}{\Delta \varepsilon}=\frac{\Delta R / R_{0}}{\Delta F} \times E_{L}=K_{F} \times E_{L}
$$

It can be seen from formula (3) that the strain sensing coefficient $K_{\varepsilon}$ can be expressed as the resistance change rateload curve slope change $\frac{\Delta R / R_{0}}{\Delta \varepsilon}$ (ie $K_{F}$ ) multiplied by the elastic modulus $\frac{\Delta F}{\Delta \varepsilon}$ (ie $E_{L}$ ). Because the elastic modulus of BFRP bars remains relatively consistent without significant damage, the slope change $K_{F}$ obtained by curve linear fitting can be used here to characterize the change of its strain sensing coefficient. It can be seen from Figure 7 that in the three loading tests, the resistance change rate-strain curve of the test piece overlaps with each other, and all can return to the initial resistance value after unloading. It shows that under the condition of repeated load-unloading, the mechanical properties and sensing performance can be kept stable, which meets the basic actual requirements.

\subsection{The Influence of Fatigue Load on Sensing Performance}

The fatigue test adopts force-controlled loading, and the loading mode is shown in Figure 7. Firstly, load the specimen to the $\left(\sigma_{\min }+\sigma_{\max }\right) / 2$ at a constant speed of $0.08 \mathrm{kN} / \mathrm{s}$, and then perform a set number of fatigue load cycles in the form of a sine wave, and the fatigue load frequency is selected as $5 \mathrm{HZ}$. 


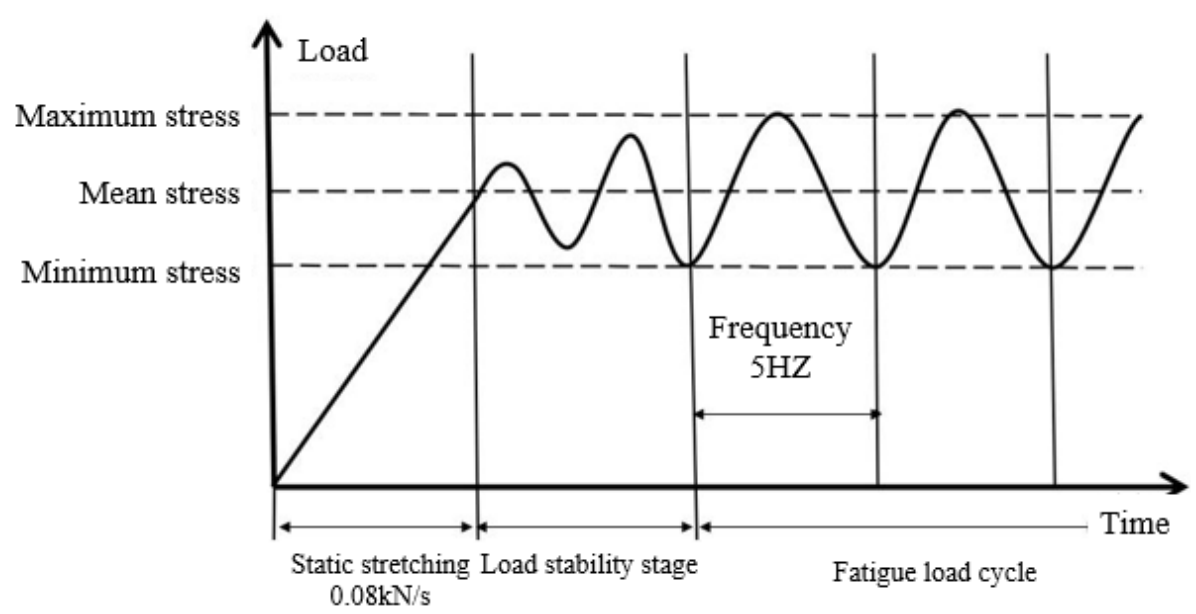

Figure 7. Fatigue test loading mode

Concerning the Chinese GB 50608 standard [8], the strength standard value $f_{k}$ with a $95 \%$ guarantee rate is selected as the design strength $f_{u}$ in the fatigue and load test. The calculation formula is:

$$
f_{k}=\mu_{f}-1.645 \sigma_{f}
$$

Here, $f_{k}$ is the design strength standard valued of $95 \%$ guarantee rate, this value is used as a reference for the load calculation in the fatigue load test design (equivalent to $f_{u}$ ). $\mu_{f}$ is the average value of tensile strength, which is $1538 \mathrm{MPa}$ from Table 1. $\sigma_{f}$ is the standard deviation of tensile strength, which is $65.91 \mathrm{MPa}$. Therefore, the strength standard value $f_{k}$ is $1429.58 \mathrm{MPa}$ obtained from equation (2).

Principle of equal strength design:

$$
f_{d}=\frac{f_{u}}{S}
$$

Here, $f_{d}$ is the design strength of FRP cable, $f_{u}$ is the standard value of the strength of FRP cable, $\mathrm{S}$ is the safety factor corresponding to the bar.

According to the FRP cable safety factor design method based on the 100-year fatigue life proposed by Feng Bo[9], the fatigue life model of the FRP bar is established, and the corresponding FRP bar safety factor designing suggestions are proposed. Among them, the designing reference value of the safety factor for the 100-year fatigue life of the BFRP bar is 3.27 , so the designing strength of the FRP cable is 437.18MPa from the formulas (5) and (6). To simulate hundreds of millions of cyclic loads on the cable in the laboratory, the method of amplifying the fatigue stress amplitude is used for research. The minimum fatigue stress $\sigma_{\min }$ in the test is a fixed value $f_{d}$, which is $0.31 f_{u}$, and the fatigue stress amplitude $\Delta \sigma$ is selected as $0.08 f_{u}, 0.1 f_{u}, 0.12 f_{u}$, respectively. The minimum fatigue stress refers to the reduction of BFRP strength by the safety factor of 100 years of fatigue life.
The no-load resistance value refers to the resistance of the self-monitoring bar under no load. As the number of fatigue cycles increase, the surface of the composite film continues to suffer fatigue damage, the no-load resistance increases, and the sensing performance also deteriorates. Under the three fatigue stress amplitude conditions, the corresponding composite film resistance was measured under no-load conditions, and the resistances of the specimens corresponding to different fatigue times were obtained as shown in Figure 8, and the cycles of fatigue failure of each specimen were shown in Table 1 . Here, $R_{0}$ is the resistance value under no-fatigue cycles and no-load, $\Delta R_{N}$ is the change in the measured value of the specimen's no-load resistance after a certain number of fatigue cycles, and $\mathrm{N}$ is the number of fatigue cycles. It can be seen from Figure 8 that in the early stage of the fatigue test (before 2000-5000 times), the resistance change rates of the specimen increase rapidly with the number of fatigue cycles. After this limit is exceeded, the growth rates of the resistance change rate gradually slow down. The number of cycles is roughly linear. In the comparison test of three specimens with different fatigue stress amplitudes, the resistance change rate does not exceed $7.29 \%$ before macro fatigue failure occurs (such as the test stage after CF-SR-8 has been subjected to 100,000 fatigue cycles). It suggests that the composite BFRP bars have stable sensing performances before the macroscopic failures of the bars occur.

Table 1. Number of load cycles corresponding to fatigue failure

\begin{tabular}{cc}
\hline Test subject & Number of load cycles \\
\hline CF-SR-12 & 40121 \\
CF-SR-10 & 72391 \\
CF-SR-8 & 125426 \\
\hline
\end{tabular}




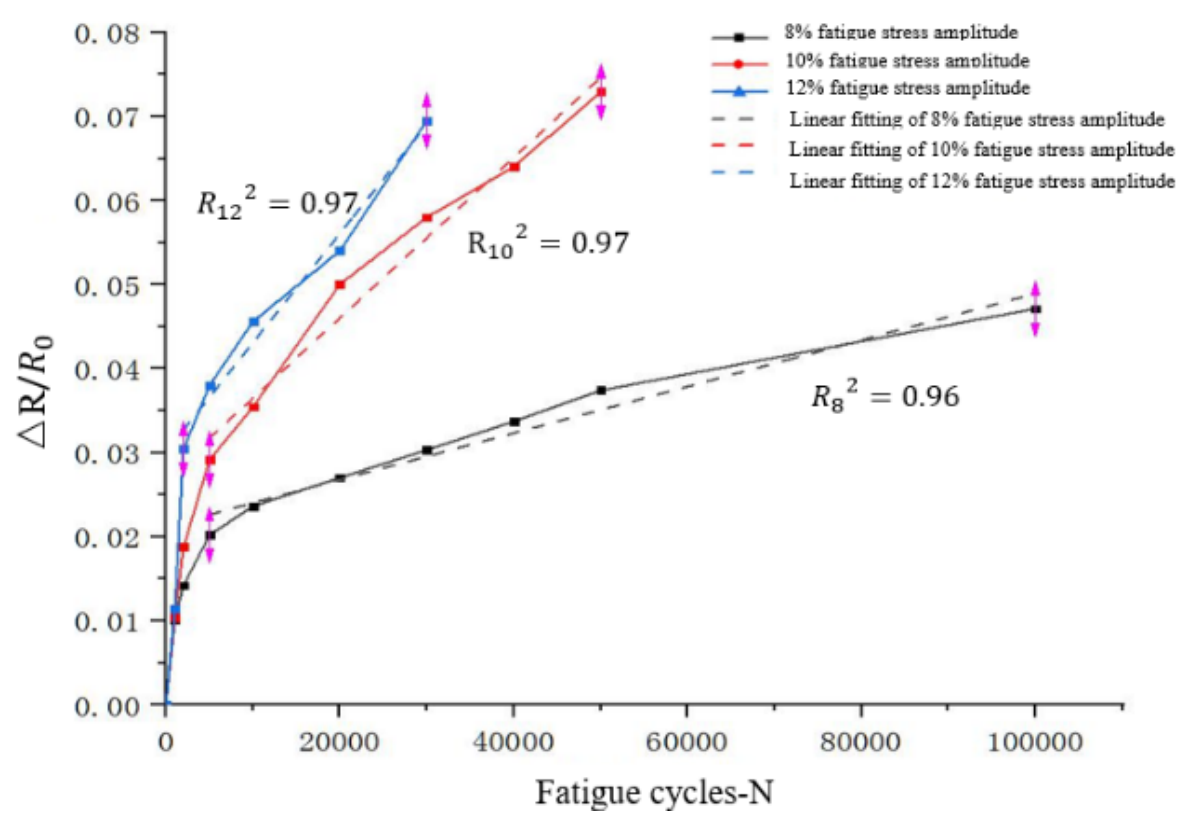

Figure 8. $\Delta R_{N} / R_{0}-\mathrm{N}$ curve under different fatigue stress amplitude

In this paper, the research on the degradation of composite film sensing performance follows the Miner linear damage theory [10].

$$
\left.\begin{array}{c}
\text { Damage definition: } D_{i}=\frac{1}{N_{i}} \\
\text { Damage accumulation: } \mathrm{D}=\sum_{i=1}^{i=n} D_{i}=\sum_{i=1}^{i=n} \frac{n_{i}}{N_{i}} \\
\text { Critical damage value: } D_{\mathrm{CR}}=1
\end{array}\right]
$$

Here, $D_{i}$ is the fatigue damage under a certain stress level $S_{i}, n_{i}$ is the corresponding number of cycles under the stress level $S_{i}, N_{i}$ is the fatigue life under the stress level $S_{i}$, and the $n_{i} / N_{i}$ is usually called the cycle ratio.

The Miner linear damage theory is applied to the fatigue degradation of the sensing performance in this test, which is the damage caused to the graphene-modified bar is equal for each load cycle, and the macroscopic performance of the fatigue damage on the bar is the change of resistance. Therefore, in theory, the resistance change and the number of load cycles should show a linear relationship. The three dashed lines in Figure 7 are the linear fitting of the curves of the specimens in the stable development stage of fatigue damage under different fatigue stress amplitudes. The formulas are:

$$
\begin{aligned}
& \triangle \varphi_{12}=1.30 \times 10^{-4} \mathrm{~N}+3.01 \\
& \triangle \varphi_{10}=9.54 \times 10^{-5} \mathrm{~N}+2.69 \\
& \triangle \varphi_{8}=2.77 \times 10^{-5} \mathrm{~N}+2.13
\end{aligned}
$$

Here, $\Delta \varphi_{12}, \Delta \varphi_{10}, \Delta \varphi_{8}$ are respectively the rates of resistance change under no-load condition after the cyclic loads, whose fatigue stress amplitudes are $12 \%, 10 \%, 8 \%$ of $f_{u} . \mathrm{N}$ is the number of load cycles. In the formula, the fitting regression coefficients R2 are $0.97,0.97$ and 0.96 . It shows that the resistance change in the stable development stage of fatigue damage has a linear relationship with the number of load cycles, which meets the Miner's linear damage theory on the assumption of sensor performance degradation.

\section{Conclusions}

Dispersing graphene nanoplatelets into epoxy was integrated with BFRP bar to serve as sensors in this study. The conclusions of the experimental results as follow:

(1) Compared with the new sensors that have been extensively researched, the graphene-modified BFRP bar has the advantage of a large range, and the strain range of graphene-modified BFRP bar.

(2) The graphene-modified BFRP bars can realize an integrated production of force and health monitoring, and have the feasibility of realizing distributed sensing at the same time.

(3) The resistance change rate of graphene-modified BFRP bars changes little during repeated tests in a wide range, which provides great convenience to construction management.

(4) In the fatigue test, under different stress amplitudes, the resistance change rate of the specimen before failure is less than $7.5 \%$, which indicated the maintained stable sensing performance.

\section{References}

[1] WANG X, SHI J Z, WU Z S. et al. Fatigue behavior of basalt fiber-reinforced polymer tendons for prestressing applications [J]. Journal of composites for construction, 2015, 20(3): OO00649.

[2] WANG X, SHI J Z, LIU J, et a1. Creep behavior of basalt fiber reinforced polymer tendons for prestressing application $[\mathrm{J}]$. Materials and Design, 2014, 59(7): 558-564.

[3] Hong W; Wu ZS, Yang CQ, et a1. Identification of modal macro - strain vector based on distributed long - gage FBG sensor under ambient vibration [C]. Sensors and Smart Structures Technologies for Civil, Mechanical, and Aerospace Systems, 2010, 7647, 7647: 36-44.

[4] Li SZ, Wu ZS. A model-flee method for damage locating and quantifying in a beam-like structure based on dynamic distributed strain measurements [J]. Computer Aided Civil and Infrastructure Engineering, 2008, 23: 404-413. 
[5] Allen, M.J., Tung, V.C. and Kaner, R.B. (2010) Honeycomb Carbon: A Review of Graphene. Chemical Reviews, 110, 132145.

[6] Novoselov, K.S., Geim, A.K., Morozov, S.V., Jiang, D., Katsnelson, M.I., Grigorieva, I.V., Dubonos, S.V. and Firsov, A.A. (2005) Two-Dimensional Gas of Massless Dirac Fermions in Graphene. Nature, 438, 197-200.

[7] Meyer, J.C., Geim, A.K., Katsnelson, M.I., et al. (2007) The Structure of Suspended Graphene Sheets. Nature, 446, 60-63.
[8] GB 50608-2010. Technical code for infrastructure application of FRP composites. (in Chinese)

[9] Feng Bo, Wang Xin, Wu ZhiShen. Fatigue lifr assessment of FRP cable for long-span cable-stayed bridge [J]. Composite Structures, 2019, 210:159-166.

[10] Miner MA. Cumulative damage in fatigue. Journal of Applied Mechanics, 1945, 12(3): 159-164. 